\title{
A Robust Tracking Controller for Dynamically Positioned Surface Vessels with Added Mass
}

\author{
Baris Bidikli, Enver Tatlicioglu*, and Erkan Zergeroglu
}

\begin{abstract}
This work concentrates on tracking control of dynamically positioned surface vessels with asymmetric added mass terms affecting the system model at the acceleration level. Specifically, we propose a novel continuous robust controller for surface vessels that, in addition to asymmetric added mass in its inertia matrix, contains unstructured uncertainties in all its system matrices. The proposed controller compensates the overall system uncertainties and ensures asymptotic tracking, while requiring only the knowledge of the sign of the leading principle minors of the input gain matrix. Lyapunov based approaches are applied in order to prove the stability of the closed-loop system and asymptotic convergence of the tracking error signal.
\end{abstract}

\section{INTRODUCTION}

Mostly because of its importance in marine industry, control of marine vehicles, especially control of slowly moving surface vessels, is a popular research topic. Operations such as towing, laying cables to the bottom of the ocean, and most operations related to the offshore oil industry usually provide a sufficiently smooth, and a slow trajectory to be tracked. Researchers/engineers are required to design controllers/autopilots to obtain satisfactory tracking performance.

Several aspects of the above control problem were investigated and can be found in the literature [1], [2], [3]. There are linear control designs such as proportional integral derivative controllers in cascade with a low-pass filter [4], optimal control laws in conjunction with Kalman filtering techniques [5], [6]. Basically, the aforementioned control formulations linearize the system dynamics about a set of pre-specified yaw angles [7]. On the other hand, there are several nonlinear robust and/or adaptive control designs that take the nonlinear ship dynamics into account in order to overcome the problems inherited by linearization [7], [8], [9], [10], [11], [12]. In [9], a class of nonlinear proportional derivative control laws for position regulation were developed without guaranteeing their robustness against parametric uncertainties, and in [11], a robust nonlinear control law using singular perturbation theory that takes parametric uncertainties and external disturbances into account was presented. Some other past research has focused on designing output feedback control algorithms [7], [8], [13], [14]. In [7], a nonlinear

B. Bidikli and E. Tatlicioglu are with the Department of Electrical \& Electronics Engineering, Izmir Institute of Technology, Izmir, 35430 Turkey (Phone: +90 (232) 7506536; Fax: +90 (232) 7506599; E-mail: [barisbidikli,enver]@iyte.edu.tr).

E. Zergeroglu is with the Department of Computer Engineering, Gebze Institute of Technology, 41400, Gebze, Kocaeli, Turkey (Email: ezerger@bilmuh.gyte.edu.tr).

E. Tatlicioglu and E. Zergeroglu are funded by The Scientific and Technological Research Council of Turkey via grant number 113E147.

*To whom all the correspondence should be addressed. output feedback controller utilizing an observer backstepping method was designed. An observer based output feedback tracking controller for fully actuated ships was presented in [14].

Review of the relevant literature highlights the fact that almost all of the past control designs considered the inertia matrix of the ship to be symmetric and positive definite, and laid their analysis and designs down on this assumption. As detailed in [2], [3], [10] and [15], during the cruise, all the flow is effected by the motion of the ship and as a result of this, vibrations with different amplitudes occur on different parts of the flow. This situation results as pressure effects and moments acting on different parts of the ship, causes additional force and has influence on the acceleration of the ship. This effect, referred as added mass, is represented in the dynamic model. There are different conventions [10], [15] on how to represent the added mass effects in the dynamic model. In [15], after using inertial velocity as the velocity state, the added mass effects are represented via the inertia matrix.

The significance of added mass effects is due to its asymmetric nature which causes the inertia matrix lose its symmetry. From a control theory perspective, the symmetric nature of the inertia matrix is extremely useful as it is utilized in a quadratic term in the Lyapunov function. And when not appropriately dealt with, this may cause reduction in performance, even instability. To our best knowledge there are only a few control design works that considered asymmetric added mass in the inertia matrix, as in robust and adaptive type controllers designed in [16] and [17], respectively. The aforementioned controllers were designed based on Lyapunov-type analysis methods, and were able to achieve only the ultimate boundedness of the tracking error signals.

In this paper, robust control of surface vessels with added mass effect is discussed. The added mass terms are considered to be affecting the system dynamics at the acceleration level (i.e., inertial velocity was chosen as the velocity state). Furthermore, the added mass effects are assumed to be asymmetrical, which results in an asymmetric inertia matrix in system dynamics. In addition, the dynamic model considered is subject to unstructured uncertainties. In the control design, the mathematical model of the ship is first converted into a compact form where neither symmetry nor positive definiteness of the input gain matrix is known. A matrix decomposition is then applied to the input gain matrix to obtain a symmetric and positive definite matrix that a filtered version of the tracking error is multiplied with. However, this 
decomposition results in the control input to be premultiplied first with a known diagonal matrix with entries +1 or -1 , and next with an uncertain unity upper triangular matrix ${ }^{1}$. A robust controller utilizing a modified version of the integral of the sign of the tracking error [18] is then proposed. The stability of the closed-loop system and the convergence of the tracking error are demonstrated via a novel analysis based on Lyapunov-type methods.

\section{System Model AND PRoperties}

The mathematical model for a dynamically positioned surface vessel is represented by [2], [3], [10]

$$
\begin{aligned}
M_{s} \dot{v}+C_{s} v+D_{s} v & =\tau \\
\dot{x} & =R v
\end{aligned}
$$

where $x(t) \triangleq\left[x_{p}, y_{p}, \psi\right]^{T} \in \mathbb{R}^{3}$ is the position vector that contains translational positions $x_{p}(t), y_{p}(t) \in \mathbb{R}$ in $\mathrm{X}$ - and $\mathrm{Y}$ - directions, respectively, and the yaw angle of the ship $\psi(t) \in \mathbb{R}, v(t)=[u, v, \dot{\psi}]^{T} \in \mathbb{R}^{3}$ is the body-fixed linear and angular velocity vector. Also in (1), $M_{s}(\psi), C_{s}\left(v, v_{r}\right)$, $D_{s}\left(v, v_{r}\right) \in \mathbb{R}^{3 \times 3}$ represent inertia matrix, centripetal and Coriolis forces, hydrodynamic damping terms, respectively, $v_{r}(t) \in \mathbb{R}^{3}$ is the relative velocity between the fluids and the vessel, and the control input torque vector is represented by $\tau(t) \in \mathbb{R}^{3}$. In (2), $R(\psi) \in S O(3)$ is the rotation matrix that has the form

$$
R(\psi)=\left[\begin{array}{lll}
\cos (\psi) & -\sin (\psi) & 0 \\
\sin (\psi) & \cos (\psi) & 0 \\
0 & 0 & 1
\end{array}\right]
$$

The inertia matrix of the ship is obtained as [2]

$$
M_{s}=M_{R B}+M_{A}
$$

where $M_{R B}(\psi) \in \mathbb{R}^{3 \times 3}$ represents the positive definite, symmetric rigid body inertia matrix and $M_{A}(\psi) \in \mathbb{R}^{3 \times 3}$ represents the added mass inertia matrix. It is noted in [2] that the inertia term due to added mass $M_{A}(\psi)$ is not necessarily symmetric. After summed with symmetric $M_{R B}(\psi)$, the inertia matrix $M_{s}(\psi)$ loses its symmetry. It is highlighted that symmetry of the inertia matrix is essential especially when it is to be used in a quadratic term in the Lyapunov function. On the other hand, it is assumed that this added mass term does not result in $M_{s}(\psi)$ losing rank [i.e., $M_{s}(\psi)$ is full rank].

In an attempt to obtain a compact representation of the mathematical model of the ship in (1) and (2), the time derivative of (2) is taken

$$
\ddot{x}=\dot{R} v+R \dot{v}
$$

which includes the time derivative of the rotation matrix that can be obtained as

$$
\dot{R}=R S_{3}
$$

\footnotetext{
${ }^{1} \mathrm{~A}$ unity upper triangular matrix is an upper triangular matrix with ones on the main diagonal.
}

where $S_{3}(\dot{\psi}) \in \mathbb{R}^{3 \times 3}$ is a skew-symmetric defined as

$$
S_{3} \triangleq\left[\begin{array}{lll}
0 & -\dot{\psi} & 0 \\
\dot{\psi} & 0 & 0 \\
0 & 0 & 0
\end{array}\right] .
$$

After substituting (6) into (5), we obtain

$$
\ddot{x}=R M_{s}^{-1} \tau-R\left[M_{s}^{-1}\left(C_{s}+D_{s}\right)-S_{3}\right] v
$$

where (1) was utilized. In order to obtain a more compact form, the right-hand side of (8) can be rewritten as

$$
\ddot{x}=h+G \tau
$$

where $h(x, \dot{x}) \in \mathbb{R}^{3}$ and $G(x, \dot{x}) \in \mathbb{R}^{3 \times 3}$ are defined as

$$
\begin{aligned}
h & \triangleq-R\left[M_{s}^{-1}\left(C_{s}+D_{s}\right)-S_{3}\right] v \\
G & \triangleq R M_{s}^{-1} .
\end{aligned}
$$

It is assumed that $G(x, \dot{x})$ is a real matrix with non-zero leading principle minors which follows from $M_{s}(\psi)$ being full rank. This allows us to utilize the following matrix decomposition [19], [20]

$$
G=S(x, \dot{x}) D U(x, \dot{x})
$$

where $S(x, \dot{x}) \in \mathbb{R}^{3 \times 3}$ is a symmetric positive definite matrix, $D \in \mathbb{R}^{3 \times 3}$ is a diagonal matrix with entries being \pm 1 , and $U(x, \dot{x}) \in \mathbb{R}^{3 \times 3}$ is a unity upper triangular matrix. We applied the above matrix decomposition to several models in the literature and $D$ came out to be an identity matrix. Despite this, we will present our derivations for the general case where we only assume that $D$ is available for control design (see [21] for the precedence of this type assumption).

From (9), it is easy to obtain

$$
\tau=G^{-1}(\ddot{x}-h)
$$

provided the assumption that the leading principal minors of $G(\cdot)$ are non-zero. After taking the time derivative of (9), substituting (12) and (13), and then performing straightforward mathematical manipulations yield

$$
\dddot{x}=\varphi+S D U \dot{\tau}
$$

where $\varphi(x, \dot{x}, \ddot{x}) \in \mathbb{R}^{3}$ is an auxiliary signal that is defined as

$$
\varphi \triangleq \dot{h}+\dot{G} G^{-1}(\ddot{x}-h) .
$$

At this point, we would like to define $M(x, \dot{x}) \in \mathbb{R}^{3 \times 3}$ as the inverse of $S(x, \dot{x})$. It is remarked that since $S(x, \dot{x})$ is symmetric and positive definite, then so is $M(x, \dot{x})$. Furthermore, $M(x, \dot{x})$ satisfies the following inequalities

$$
\underline{m}\|\chi\|^{2} \leq \chi^{T} M(x, \dot{x}) \chi \leq \bar{m}(x, \dot{x})\|\chi\|^{2} \quad \forall \chi \in \mathbb{R}^{3 \times 1}
$$

where $\underline{m} \in \mathbb{R}$ and $\bar{m}(x, \dot{x}) \in \mathbb{R}$ represent a positive bounding constant and a positive non-decreasing function, respectively.

Multiplying both sides of (14) with $M(x, \dot{x})$ results in

$$
M \dddot{x}=f+D U \dot{\tau}
$$

where $f(x, \dot{x}, \ddot{x}) \triangleq M \varphi \in \mathbb{R}^{3}$. 


\section{ERRor SyStEM DEVELOPMENT}

Our main control objective is to ensure that the translational positions and the yaw angle would track a given reference trajectory while, at the same time, ensuring the boundedness of all the signals under the closed-loop operation. The control design is based on availability of $x(t)$ and $\dot{x}(t)$ (i.e., full-state feedback).

In order to quantify the tracking control objective, the output tracking error, $e_{1}(t) \in \mathbb{R}^{3}$, is defined as the difference between the reference trajectory and the position of the ship as

$$
e_{1} \triangleq x_{d}-x
$$

where $x_{d}(t) \in \mathbb{R}^{3}$ is the reference trajectory chosen smooth enough in the sense that

$$
x_{d}(t) \in \mathcal{C}^{3} \text { and } x_{d}^{(i)}(t) \in \mathcal{L}_{\infty}, i=0,1,2,3 .
$$

In order to obtain a first order stability analysis (i.e., only first order time derivatives to appear in the time derivative of the Lyapunov function), an auxiliary error signal, denoted by $e_{2}(t) \in \mathbb{R}^{3}$, and a filtered error term, denoted by $r(t) \in \mathbb{R}^{3}$, are defined as follows

$$
\begin{aligned}
e_{2} & \triangleq \dot{e}_{1}+e_{1} \\
r & \triangleq \dot{e}_{2}+\alpha e_{2}
\end{aligned}
$$

where $\alpha \in \mathbb{R}^{3 \times 3}$ is a positive definite, diagonal, constant gain matrix. After premultiplying the time derivative of (21) with $M(\cdot)$, the following expression can be obtained

$$
M \dot{r}=M\left(\dddot{x}_{d}+\ddot{e}_{1}+\alpha \dot{e}_{2}\right)-f-D U \dot{\tau}
$$

where (17), (18) and (20) were utilized. To obtain a compact form for the right-hand side of (22), we define an auxiliary function $N\left(x, \dot{x}, \ddot{x}, x_{d}, \dot{x}_{d}, \ddot{x}_{d}, \dddot{x}_{d}, t\right) \in \mathbb{R}^{3}$ as follows

$$
N \triangleq M\left(\dddot{x}_{d}+\ddot{e}_{1}+\alpha \dot{e}_{2}\right)-f+e_{2}+\frac{1}{2} \dot{M} r .
$$

In view of (23), the expression in (22) can now be reformulated as

$$
M \dot{r}=-\frac{1}{2} \dot{M} r-e_{2}-D U \dot{\tau}+N .
$$

In the right-hand side of (24), [via the definition of $N(\cdot)$ in (23)] the terms $-\frac{1}{2} \dot{M} r$ and $-e_{2}$ are introduced to cancel some terms that will appear in the time derivative of the subsequently designed Lyapunov function.

The auxiliary function $N(\cdot)$ is now segregated into two auxiliary functions in a way by grouping the terms that can be bounded by known constants, and the terms that can be bounded by error signals. Mathematically, $\bar{N}(t), \widetilde{N}(t) \in \mathbb{R}^{3}$ are defined as

$$
\begin{aligned}
\bar{N} & \left.\triangleq N\right|_{x=x_{d}, \dot{x}=\dot{x}_{d}, \ddot{x}=\ddot{x}_{d}} \\
\widetilde{N} & \triangleq N-\bar{N} .
\end{aligned}
$$

Finally, after substituting the above definitions, the error dynamics in (24) can be rearranged as

$$
M \dot{r}=-\frac{1}{2} \dot{M} r-e_{2}-D U \dot{\tau}+\tilde{N}+\bar{N} .
$$

\section{CONTROller Formulation}

Based on the open-loop error system in (27) and the subsequent stability analysis, the control input $\tau(t)$ is designed to have the following form

$$
\tau=D K\left[e_{2}(t)-e_{2}\left(t_{0}\right)+\alpha \int_{t_{0}}^{t} e_{2}(\sigma) d \sigma\right]+D \Pi
$$

where the auxiliary signal $\Pi(t) \in \mathbb{R}^{3}$ is generated according to the update law

$$
\dot{\Pi}(t)=C \operatorname{Sgn}\left(e_{2}(t)\right) \text { with } \Pi\left(t_{0}\right)=0_{3} .
$$

In (28) and (29), $K, C \in \mathbb{R}^{3 \times 3}$ are positive definite, diagonal, constant gain matrices, $0_{3} \in \mathbb{R}^{3}$ is a vector of zeros and $\operatorname{Sgn}(\cdot) \in \mathbb{R}^{3}$ is the vector signum function. The open-loop error dynamics in (27) requires the time derivative of the control input. By using the structures of (28) and (29), the time derivative of control input can be obtained in the following form

$$
\dot{\tau}=D K r+D C \operatorname{Sgn}\left(e_{2}\right)
$$

where (21) was also utilized. The control gain is chosen as $K=I_{3}+k_{p} I_{3}+\operatorname{diag}\left\{k_{d, 1}, k_{d, 2}, 0\right\}$ where $k_{p}, k_{d, 1}, k_{d, 2} \in \mathbb{R}$ are positive, constant controller gains, the notation diag $\{\cdot\}$ represents a diagonal matrix, and $I_{3} \in \mathbb{R}^{3 \times 3}$ is the standard identity matrix. Finally, after substituting (30) into (27) and then adding and subtracting $D K r(t)$, following closed-loop error system for $r(t)$ is obtained

$$
\begin{aligned}
M \dot{r}= & -\frac{1}{2} \dot{M} r-e_{2}-K r+\tilde{N}+\bar{N} \\
& -D\left(U-I_{3}\right) D K r-D U D C \operatorname{Sgn}\left(e_{2}\right)
\end{aligned}
$$

where the fact that $D D=I_{3}$ was utilized.

We would like to draw attention to the last two terms of (31) by investigating them separately before proceeding with the stability analysis. It is highlighted that, our investigation on these two terms rely on the fact that $U(\cdot)$ is a unity upper triangular matrix and thus $\left(U-I_{3}\right)$ is a strictly upper triangular matrix.

Notice that, we can rewrite $D\left(U-I_{3}\right) D K r$ as follows

$$
D\left(U-I_{3}\right) D K r=\left[\begin{array}{c}
\Lambda_{1} \\
\Lambda_{2} \\
0
\end{array}\right]+\left[\begin{array}{c}
\Phi_{1} \\
\Phi_{2} \\
0
\end{array}\right]
$$

where $\Lambda_{1}(t), \Lambda_{2}(t), \Phi_{1}(t), \Phi_{2}(t) \in \mathbb{R}$ are auxiliary signals being defined as

$$
\begin{aligned}
& \Lambda_{1} \triangleq d_{1} d_{2} k_{2} \widetilde{U}_{1,2} r_{2}+d_{1} d_{3} k_{3} \widetilde{U}_{1,3} r_{3} \\
& \Lambda_{2} \triangleq d_{2} d_{3} k_{3} \widetilde{U}_{2,3} r_{3} \\
& \Phi_{1} \triangleq d_{1} d_{2} k_{2} \bar{U}_{1,2} r_{2}+d_{1} d_{3} k_{3} \bar{U}_{1,3} r_{3} \\
& \Phi_{2} \triangleq d_{2} d_{3} k_{3} \bar{U}_{2,3} r_{3}
\end{aligned}
$$

with $\bar{U}_{1,2}(t), \bar{U}_{1,3}(t), \bar{U}_{2,3}(t), \widetilde{U}_{1,2}(t), \widetilde{U}_{1,3}(t), \widetilde{U}_{2,3}(t) \in$ $\mathbb{R}$ are defined as

$$
\begin{aligned}
\bar{U}_{i, j} & \left.\triangleq U_{i, j}\right|_{x=x_{d}, \dot{x}=\dot{x}_{d}} \\
\widetilde{U}_{i, j} & \triangleq U_{i, j}-\bar{U}_{i, j}
\end{aligned}
$$


where $U_{i, j}(x, \dot{x}) \in \mathbb{R}$ are the entries of $U(x, \dot{x})$. Notice from (34) that $\Lambda_{2}(t)$ depends on $k_{3}$, and from (33), $\Lambda_{1}(t)$ depends on $k_{3}$ and $k_{2}$. Similarly, from (36), $\Phi_{2}(t)$ depends on $k_{3}$, and from (35), $\Phi_{1}(t)$ depends on $k_{3}$ and $k_{2}$.

On the other hand, the term $D U D C \operatorname{Sgn}\left(e_{2}\right)$ can be decomposed as

$$
\operatorname{DUDCSgn}\left(e_{2}\right)=\left[\Psi^{T}, 0\right]^{T}+\Theta
$$

with two auxiliary signals $\Psi(t) \in \mathbb{R}^{2}$ and $\Theta(t) \in \mathbb{R}^{3}$ are defined as

$$
\begin{aligned}
{\left[\Psi^{T}, 0\right]^{T} } & =D(U-\bar{U}) D C \operatorname{Sgn}\left(e_{2}\right) \\
\Theta & \triangleq D \bar{U} D C \operatorname{Sgn}\left(e_{2}\right)
\end{aligned}
$$

where $\left.\bar{U}\left(x_{d}, \dot{x}_{d}\right) \triangleq U\right|_{x=x_{d}, \dot{x}=\dot{x}_{d}} \in \mathbb{R}^{3 \times 3}$ is a function of reference trajectory and its time derivative, and $\Psi_{i}(t) \in \mathbb{R}$, $i=1,2$ and $\Theta_{i}(t) \in \mathbb{R}, i=1,2,3$, are defined as

$$
\begin{aligned}
\Psi_{i} & =d_{i} \sum_{j=i+1}^{3} d_{j} C_{j} \widetilde{U}_{i, j} \operatorname{sgn}\left(e_{2, j}\right) \\
\Theta_{i} & =d_{i} \sum_{j=i}^{3} d_{j} C_{j} \bar{U}_{i, j} \operatorname{sgn}\left(e_{2, j}\right) .
\end{aligned}
$$

Remark 1: The Mean Value Theorem [22] can be utilized to develop the following upper bounds

$$
\begin{aligned}
\|\widetilde{N}(\cdot)\| & \leq \rho_{\widetilde{N}}(\|z\|)\|z\| \\
\left\|\widetilde{U}_{i, j}(\cdot)\right\| & \leq \rho_{i, j}(\|z\|)\|z\|
\end{aligned}
$$

where $\rho_{\widetilde{N}}(\cdot), \rho_{i, j}(\cdot) \in \mathbb{R}$ are non-negative, globally invertible, non-decreasing functions of their arguments, and $z(t) \in \mathbb{R}^{9}$ is defined by

$$
z \triangleq\left[\begin{array}{lll}
e_{1}^{T} & e_{2}^{T} & r^{T}
\end{array}\right]^{T} .
$$

In view of (19) (i.e., the smoothness of the reference trajectory), it can be seen from (25) and (37) that $\bar{N}(t)$ and $\bar{U}_{i, j}(t)$ can be bounded by known constants in the sense that [18]

$$
\begin{aligned}
\left|\bar{N}_{i}(t)\right| & \leq \zeta_{\bar{N}_{i}} \\
\left|\bar{U}_{i, j}(t)\right| & \leq \zeta_{\bar{U}_{i, j}}
\end{aligned}
$$

where $\zeta_{\bar{N}_{i}}, \zeta_{\bar{U}_{i, j}} \in \mathbb{R}$ are positive bounding constants. Based on (33)-(36), (42), (43), following upper bounds can be obtained for $i=1,2$

$$
\begin{aligned}
\left|\Lambda_{i}\right| & \leq \sum_{j=i+1}^{3} k_{j} \rho_{i, j}(\|z\|)\|z\|\left|r_{j}\right| \leq \rho_{\Lambda_{i}}(\|z\|)\|z\|(49) \\
\left|\Phi_{i}\right| & \leq \sum_{j=i+1}^{3} k_{j} \zeta_{\bar{U}_{i, j}}\left|r_{j}\right| \leq \zeta_{\Phi_{i}}\|z\| \\
\left|\Psi_{i}\right| & \leq \sum_{j=i+1}^{3} C_{j} \rho_{i, j}(\|z\|)\|z\| \leq \rho_{\Psi_{i}}(\|z\|)\|z\|
\end{aligned}
$$

and for $i=1,2,3$

$$
\left|\Theta_{i}\right| \leq \sum_{j=i}^{3} C_{j} \zeta_{\bar{U}_{i, j}} \leq \zeta_{\Theta_{i}}
$$

where (44)-(48) were utilized. From (52), it is easy to see that $\|\Theta\| \leq \zeta_{\Theta}$ is satisfied for some positive bounding constant $\zeta_{\Theta} \in \mathbb{R}$, and from (49)-(51), we have

$$
\left|\Lambda_{i}\right|+\left|\Phi_{i}\right|+\left|\Psi_{i}\right| \leq \rho_{i}(\|z\|)\|z\|
$$

where $\rho_{i}(\|z\|) \in \mathbb{R} i=1,2$, are non-negative, globally invertible, non-decreasing functions satisfying

$$
\rho_{\Lambda_{i}}+\rho_{\Psi_{i}}+\zeta_{\Phi_{i}} \leq \rho_{i} .
$$

At this point, we are now ready to continue with the stability analysis of the proposed robust controller.

\section{Stability Analysis}

In this section ${ }^{2}$, initially, the boundedness of the error signals under the closed-loop operation will be proven by using a Lyapunov type analysis. Then, a lemma will be presented and an upper bound for the integral of the absolute values of the entries of the time derivative of $e_{2}(t)$ will be obtained by using the boundedness result. In order to prove the non-negativity of a Lyapunov-like function, this upper bound will be utilized in another lemma that will be used in our final analysis which proves asymptotic stability of the overall closed-loop system.

Theorem 1: (Boundedness proof) For the mathematical model of the ship in (1) and (2), the controller in (28) and (29) guarantee the boundedness of the error signals in (18), (20) and (21) provided that the controller gains $k_{d, 1}, k_{d, 2}$ and $k_{p}$ are chosen large enough compared to the initial conditions of the system and the following condition is satisfied

$$
\lambda_{\min }(\alpha) \geq \frac{1}{2}
$$

where the notation $\lambda_{\min }(\alpha)$ denotes the minimum eigenvalue of the gain matrix $\alpha$.

Proof: Due to page limitations, only a highlight of the proof is provided. The reader is referred to [23] for a similar proof. The non-negative function $V_{1}(z) \in \mathbb{R}$ is defined as

$$
V_{1} \triangleq \frac{1}{2} e_{1}^{T} e_{1}+\frac{1}{2} e_{2}^{T} e_{2}+\frac{1}{2} r^{T} M r
$$

In view of (16), the Lyapunov function in (56) can be lower and upper bounded in the following manner

$$
\frac{1}{2} \min \{1, \underline{m}\}\|z\|^{2} \leq V_{1}(z) \leq \frac{1}{2} \max \{1, \bar{m}(\|z\|)\}\|z\|^{2}
$$

where $z(t)$ was defined in (46), and the terms $\underline{m}, \bar{m}(\|z\|)$ were introduced in (16). Taking the time derivative of (56), making necessary substitutions, and then performing straightforward mathematical manipulations and grouping, results in the following inequality

$$
\dot{V}_{1} \leq-\beta_{1} V_{1}+\delta \varepsilon^{2}
$$

where $\beta_{1} \in \mathbb{R}$ is a positive constant. From (56), and (58), we can conclude that $V_{1}(t) \in \mathcal{L}_{\infty}$, therefore $e_{1}(t), e_{2}(t)$ and $r(t)$ are uniformly ultimately bounded. Standard signal

\footnotetext{
${ }^{2}$ Due to the page limitations, some parts of the stability analysis are referred to the technical report [23] by the authors.
} 
chasing arguments can then be utilized to prove that all the signals remain bounded under the closed-loop operation.

Lemma 1: Provided that $e_{2}(t)$ and $\dot{e}_{2}(t)$ are bounded, the following expression for the upper bound of the integral of the absolute value of the $i$-th entry of $\dot{e}_{2}(t) i=1,2,3$ can be obtained

$$
\int_{t_{0}}^{t}\left|\dot{e}_{2, i}(\sigma)\right| d \sigma \leq \gamma_{1}+\gamma_{2} \int_{t_{0}}^{t}\left|e_{2, i}(\sigma)\right| d \sigma+\left|e_{2, i}\right|
$$

where $\gamma_{1}, \gamma_{2} \in \mathbb{R}$ are some positive bounding constants.

Proof: The proof is similar to that of the one given in [24], it can also be found in Appendix B of [23].

Following decomposition is essential for the proof of Lemma 2.

Remark 2: Notice that, as a result of the fact that $\bar{U}(t)$ being unity upper triangular, $\Theta(t)$ in (41) can be rewritten as

$$
\Theta=\left(I_{3}+\Omega\right) C \operatorname{Sgn}\left(e_{2}\right)
$$

where $\Omega(t) \triangleq D\left(\bar{U}-I_{3}\right) D \in \mathbb{R}^{3 \times 3}$ is a strictly upper triangular matrix. Since it is a function of the reference trajectory and its time derivatives, its entries, denoted by $\Omega_{i, j}(t) \in \mathbb{R}$, are bounded in the sense that

$$
\left|\Omega_{i, j}\right| \leq \zeta_{\Omega_{i, j}}
$$

where $\zeta_{\Omega_{i, j}} \in \mathbb{R}$ are positive bounding constants.

Lemma 2: Consider the term

$$
L \triangleq r^{T}\left(\bar{N}-\left(I_{3}+\Omega\right) C \operatorname{Sgn}\left(e_{2}\right)\right) .
$$

Provided that the entries of the control gain matrix $C$ are chosen to satisfy

$$
\begin{aligned}
& C_{3} \geq \zeta_{\bar{N}_{3}}\left(1+\frac{\gamma_{2}}{\alpha_{3}}\right) \\
& C_{2} \geq\left(\zeta_{\bar{N}_{2}}+\zeta_{\Omega_{2,3}} C_{3}\right)\left(1+\frac{\gamma_{2}}{\alpha_{2}}\right) \\
& C_{1} \geq\left(\zeta_{\bar{N}_{1}}+\zeta_{\Omega_{1,2}} C_{2}+\zeta_{\Omega_{1,3}} C_{3}\right)\left(1+\frac{\gamma_{2}}{\alpha_{1}}\right)
\end{aligned}
$$

in order, then it can be concluded that

$$
\int_{t_{0}}^{t} L(\sigma) d \sigma \leq \zeta_{L}
$$

where $\zeta_{L} \in \mathbb{R}$ is a positive bounding constant defined as

$$
\zeta_{L} \triangleq \gamma_{1} \sum_{i=1}^{2} \sum_{j=i+1}^{3} \zeta_{\Omega_{i, j}} C_{j}+\gamma_{1} \sum_{i=1}^{3} \zeta_{\bar{N}_{i}}+\sum_{i=1}^{3} C_{i}\left|e_{2, i}\left(t_{0}\right)\right|
$$

Proof: See Appendix C of [23].

Theorem 2: (Asymptotic convergence proof) Given the ship dynamic model in (1) and (2), the continuous robust controller of (28) and (29) ensures the tracking error signal $e_{1}(t)$ and the auxiliary error signals $e_{2}(t)$ and $r(t)$ converge to zero asymptotically in the sense that

$$
\left\|e_{1}(t)\right\|,\left\|e_{2}(t)\right\|,\|r(t)\| \rightarrow 0 \text { as } t \rightarrow+\infty
$$

provided that $\alpha$ is chosen to satisfy (55), the entries of $C$ are chosen to satisfy (63)-(65), and $k_{p}, k_{d, 1}, k_{d, 2}$ are chosen large enough.
Proof: Let the auxiliary function $P(t) \in \mathbb{R}$ be defined as follows

$$
P \triangleq \zeta_{L}-\int_{t_{0}}^{t} L(\sigma) d \sigma
$$

where the terms $\zeta_{L}$ and $L(t)$ were defined in (67) and (62), respectively. When the entries of the control gain matrix $C$ are chosen to satisfy (63)-(65), from the proof of Lemma 2 given in Appendix $\mathrm{C}$ of [23], we can conclude that $P(t)$ is non-negative.

At this stage, consider the Lyapunov function $V(s, t) \in \mathbb{R}$ defined as

$$
V \triangleq V_{1}+P
$$

where $s(t) \in \mathbb{R}^{10}$ is defined as

$$
s \triangleq\left[\begin{array}{ll}
z^{T} & \sqrt{P}
\end{array}\right]^{T}
$$

and $V_{1}(t) \in \mathbb{R}$ was defined in (56). By utilizing (16), the Lyapunov function in (70) can be lower and upper bounded in the following form

$$
W_{1}(s) \leq V(s, t) \leq W_{2}(s)
$$

where $W_{1}(s), W_{2}(s) \in \mathbb{R}$ are defined as

$$
W_{1} \triangleq \lambda_{2}\|s\|^{2}, W_{2} \triangleq \lambda_{3}(\|s\|)\|s\|^{2}
$$

with $\lambda_{2} \triangleq \frac{1}{2} \min \{1, \underline{m}\}$ and $\lambda_{3} \triangleq \max \left\{1, \frac{1}{2} \bar{m}(\|z\|)\right\}$.

Taking the time derivative of $V(t)$, utilizing the time derivative of (66), cancelling common terms and following similar steps to that of proof of Theorem 1 yields

$$
\begin{aligned}
\dot{V}= & -e_{1}^{T} e_{1}+e_{1}^{T} e_{2}-e_{2}^{T} \alpha e_{2}-r^{T} r \\
+ & {\left[r^{T} \tilde{N}-k_{p} r^{T} r\right] } \\
+ & {\left[-\sum_{i=1}^{m-1} r_{i}\left(\Lambda_{i}+\Psi_{i}+\Phi_{i}\right)-\sum_{i=1}^{2} k_{d, i} r_{i}^{2}\right] }
\end{aligned}
$$

which can be rearranged to have the following form

$$
\begin{aligned}
\dot{V} \leq & -\frac{1}{2}\left\|e_{1}\right\|^{2}-\left(\lambda_{\min }(\alpha)-\frac{1}{2}\right)\left\|e_{2}\right\|^{2}-r^{T} r \\
& +\frac{\rho_{\widetilde{N}}^{2}(\|z\|)}{4 k_{p}}\|z\|^{2}+\sum_{i=1}^{2} \frac{\rho_{i}^{2}(\|z\|)}{4 k_{d, i}}\|z\|^{2} \\
\leq & -\left(\lambda_{4}-\frac{\rho_{\widetilde{N}}^{2}(\|z\|)}{4 k_{p}}-\sum_{i=1}^{2} \frac{\rho_{i}^{2}(\|z\|)}{4 k_{d, i}}\right)\|z\|^{2}
\end{aligned}
$$

where $\lambda_{4} \triangleq \min \left\{\frac{1}{2}, \lambda_{\min }(\alpha)-\frac{1}{2}\right\}$. When the controller gains $k_{p}, k_{d, 1}, k_{d, 2}$ are selected large enough such that the regions defined by $\mathcal{D}_{z} \triangleq\{z:\|z\| \leq \mathcal{R}\}$ and $\mathcal{D}_{s} \triangleq$ $\{s:\|s\| \leq \mathcal{R}\}$ with $\mathcal{R}$ being defined as

$$
\mathcal{R}=\min \left\{\rho_{\widetilde{N}}^{-1}\left(2 \sqrt{k_{p} \frac{1-\beta}{3}}\right), \rho_{i}^{-1}\left(2 \sqrt{k_{d, i} \frac{1-\beta}{3}}\right)\right\}
$$

for $i=1,2$, are non-empty, from (76) and the definition of $s$, one can restate

$$
\dot{V} \leq-\beta\|z\|^{2}=-W(s) \forall s \in \mathcal{D}_{s}
$$

where $\beta \in \mathbb{R}$ is a positive constant that satisfies $0 \leq \beta<$ 1. From the definition of (70) and (78), it is obvious that 
$V(t) \in \mathcal{L}_{\infty}$, also from the proof of Theorem 1 and outcome of standart linear analysis methods, we can conclude that all the signals in the closed-loop error system are bounded and furthermore, from the boundedness of $\dot{W}(s)$, we can state $W(s)$ is uniformly continuous. Based on the definition of $\mathcal{D}_{s}$, another region, $\mathcal{S}$, can be defined in the following form

$$
\begin{aligned}
\mathcal{S} \triangleq & \left\{s \in \mathcal{D}_{s}: W_{2}(s)<\lambda_{3}\left(\rho_{\widetilde{N}}^{-1}\left(2 \sqrt{k_{p} \frac{1-\beta}{3}}\right)\right)^{2}\right\} \\
& \cap\left\{s \in \mathcal{D}_{s}: W_{2}(s)<\lambda_{3}\left(\rho_{1}^{-1}\left(2 \sqrt{k_{d, 1} \frac{1-\beta}{3}}\right)\right)^{2}\right\} \\
& \cap\left\{s \in \mathcal{D}_{s}: W_{2}(s)<\lambda_{3}\left(\rho_{2}^{-1}\left(2 \sqrt{k_{d, 2} \frac{1-\beta}{3}}\right)\right)^{2}\right\}
\end{aligned}
$$

A direct application of Theorem 8.4 in [22] can be used to prove that $\|z(t)\| \rightarrow 0$ as $t \rightarrow+\infty \forall s\left(t_{0}\right) \in \mathcal{S}$. Based on the definition of $z(t)$, it is easy to show that $\left\|e_{1}(t)\right\|$, $\left\|e_{2}(t)\right\|,\|r(t)\| \rightarrow 0$ as $t \rightarrow+\infty \forall s\left(t_{0}\right) \in \mathcal{S}$. Note that the region of attraction can be made arbitrarily large to include any initial conditions by choosing the controller gains $k_{p}, k_{d, 1}$ and $k_{d, 2}$. This fact implies that the stability result obtained by proposed method is semi-global.

Remark 3: The control gain matrices $C$ and $K$ are required to be chosen greater than the bounds of the uncertainties. To address this issue, in [25] and [26], we designed a self-tuning algorithm for the similar type of the controllers in this paper. As a result, the controller gains in this paper are obtained via the self-tuning algorithm in [25] and [26], thus avoiding the knowledge of bounds of the uncertainties.

\section{CONCLUSION}

Robust control of dynamically positioned surface vehicles is addressed. In addition to the uncertainties in the system dynamics, added mass terms are considered to be affecting the system at the acceleration level. As a result, the inertia matrix is considered may not be symmetric due to the asymmetric added mass effects. A continuous nonlinear robust controller, that compensates the dynamical uncertainties and the asymmetry in the inertia matrix have been proposed when the input gain matrix has non-zero leading principle minors. We were able to obtain semi-global asymptotic tracking by using Lyapunov based arguments in conjunction with an integral inequality. To our best knowledge, prior this paper, the closest work that considered dynamic uncertainties and added mass effects for dynamically positioned surface vessels were [16] and [17] which achieved ultimate boundedness of the tracking error signals. This paper extends the stability result presented in [16] and [17] to asymptotic stability. That is the convergence of the tracking error signals to the origin are guaranteed as opposed to the convergence to an ultimate bound around zero.

\section{REFERENCES}

[1] T. I. Fossen, Handbook of Marine Craft Hydrodynamics and Motion Control. Hoboken, NJ, USA: John Wiley \& Sons, 2011.

[2] _ - Guidance and Control of Ocean Vehicles. New York, NY, USA: John Wiley and Sons, 1994.

[3] —-, Marine Control Systems: Guidance, Navigation, and Control of Ships, Rigs and Underwater Vehicles. Trondheim, Norway: Marine Cybernetics AS, 2002.
[4] J. G. Balchen, N. A. Jenssen, and S. Sællid, "Dynamic positioning of floating vessels based on kalman filtering and optimal control," in IFAC/IFIP Symp. on Aut. in Offshore Oil Field Operation, Amsterdam, the Netherlands, 1976, pp. 183-186.

[5] M. J. Grimble, R. J. Patton, and D. A. Wise, "The design of dynamic positioning control systems using stochastic optimal control theory," Optimal Control Applications \& Methods, vol. 1, no. 1, pp. 167-202, 1980

[6] A. J. Sørensen, S. I. Sagatun, and T. I. Fossen, "Design of a dynamic positioning system using model-based control," Control Engineering Practice, vol. 4, no. 3, pp. 359-368, 1996.

[7] T. I. Fossen and Å. Grøvlen, "Nonlinear output feedback control of dynamically positioned ships using vectorial observer backstepping," IEEE Trans. Control Syst. Technol., vol. 6, no. 1, pp. 121-128, 1998.

[8] Y. Fang, E. Zergeroglu, M. S. de Queiroz, and D. M. Dawson, "Global output feedback control of dynamically positioned surface vessels: An adaptive control approach," Mechatronics, vol. 14, no. 4, pp. 341-356, 2004.

[9] O. Fjellstad and T. I. Fossen, "Quaternion feedback regulation of underwater vehicles," in Proc. of IEEE Int. Conf. on Control Applications, Glasgow, Scotland, 1994, pp. 857-862.

[10] R. Skjetne, O. N. Smogeli, and T. I. Fossen, "A nonlinear ship manoeuvering model: Identification and adaptive control with experiments for a model ship," Modeling, Identification and Control, vol. 25, no. 1, pp. 3-27, 2004.

[11] C. C. D. Wit, E. Olguin, D. Perrier, and M. Perrier, "Robust nonlinear control of an underwater vehicle/manipulator system with composite dynamics," in Proc. IEEE Int. Conf. Robot. Autom., Leuven, Belgium, 1998, pp. 452-457.

[12] _ _ "Robust nonlinear control of an underwater vehicle/manipulator system with composite dynamics," in Proc. IEEE Int. Conf. Robot. Autom., Leuven, Belgium, 1998, pp. 452-457.

[13] B. Bidikli, E. Tatlicioglu, and E. Zergeroglu, "Observer based output feedback tracking control of dynamically positioned surface vessels," in Proc. American Control Conf., Washington, WA, USA, 2013, pp. 554-559.

[14] M. Wondergem, E. Lefeber, K. Y. Pettersen, and H. Nijmeijer, "Output feedback tracking of ships," IEEE Trans. Control Syst. Technol., vol. 19, no. 2, pp. 442-448, 2011.

[15] T. I. Fossen and J. P. Strand, "Passive nonlinear design for ships using Lyapunov methods: full-scale experiments with a supply vessel," Automatica, vol. 35, no. 1, pp. 3-16, 1999.

[16] D. Lee, E. Tatlicioglu, T. C. Burg, and D. M. Dawson, "Robust output tracking control of a surface vessel," in Proc. American Control Conf., Seattle, WA, USA, 2008, pp. 544-549.

[17] - "Adaptive output tracking control of a surface vessel," in Proc. IEEE Int. Conf. Decision and Control, Cancun, Mexico, 2008, pp. 1352-1357.

[18] B. Xian, D. M. Dawson, M. S. de Queiroz, and J. Chen, "A continuous asymptotic tracking control strategy for uncertain nonlinear systems," IEEE Trans. Autom. Control, vol. 49, no. 7, pp. 1206-1211, 2004.

[19] R. R. Costa, L. Hsu, A. K. Imai, and P. Kokotovic, "Lyapunov-based adaptive control of MIMO systems," Automatica, vol. 39, no. 7, pp. 1251-1257, 2003.

[20] A. S. Morse, "A gain matrix decomposition and some of its applications," Systems \& Control Letters, vol. 21, no. 1, pp. 1-10, 1993.

[21] J. Chen, A. Behal, and D. M. Dawson, "Robust feedback control for a class of uncertain MIMO nonlinear systems," IEEE Trans. Autom. Control, vol. 53, no. 2, pp. 591-596, 2008.

[22] H. K. Khalil, Nonlinear Systems, 3rd Edition. New York, NY, USA Prentice Hall, 2002.

[23] B. Bidikli, E. Tatlicioglu, E. Zergeroglu, and A. Bayrak, "An asymptotically stable continuous robust controller for a class of uncertain MIMO nonlinear systems," ArXiv e-prints, Tech. Rep. 1301.5483, Jan. 2013.

[24] V. Stepanyan and A. Kurdila, "Asymptotic tracking of uncertain systems with continuous control using adaptive bounding," IEEE Trans. Neural Netw., vol. 20, no. 8, pp. 1320-1329, 2009.

[25] B. Bidikli, E. Tatlicioglu, A. Bayrak, and E. Zergeroglu, "A new robust 'integral of sign of error' feedback controller with adaptive compensation gain," in Proc. IEEE Int. Conf. Decision and Control, Florence, Italy, 2013, pp. 3782-3787.

[26] B. Bidikli, E. Tatlicioglu, and E. Zergeroglu, "A self tuning RISE controller formulation," in Proc. American Control Conf., Portland, OR, USA, 2014, pp. 5608-5613. 